\title{
Over-expression of inositol 1,4,5-trisphosphate receptor type 3 in human endometrial cancer.
}

Shahan Mamoor ( $\square$ shahanmamoor@gmail.com )

https://orcid.org/0000-0003-4150-0936

\section{Short Report}

Keywords: endometrial cancer, gynecologic cancers, endometrium, ITPR3, inositol 1,4,5-trisphosphate receptor type 3 , systems biology of endometrial cancer, targeted therapeutics in endometrial cancer.

Posted Date: June 3rd, 2021

DOI: https://doi.org/10.21203/rs.3.rs-582011/v1

License: (c) (i) This work is licensed under a Creative Commons Attribution 4.0 International License. Read Full License 


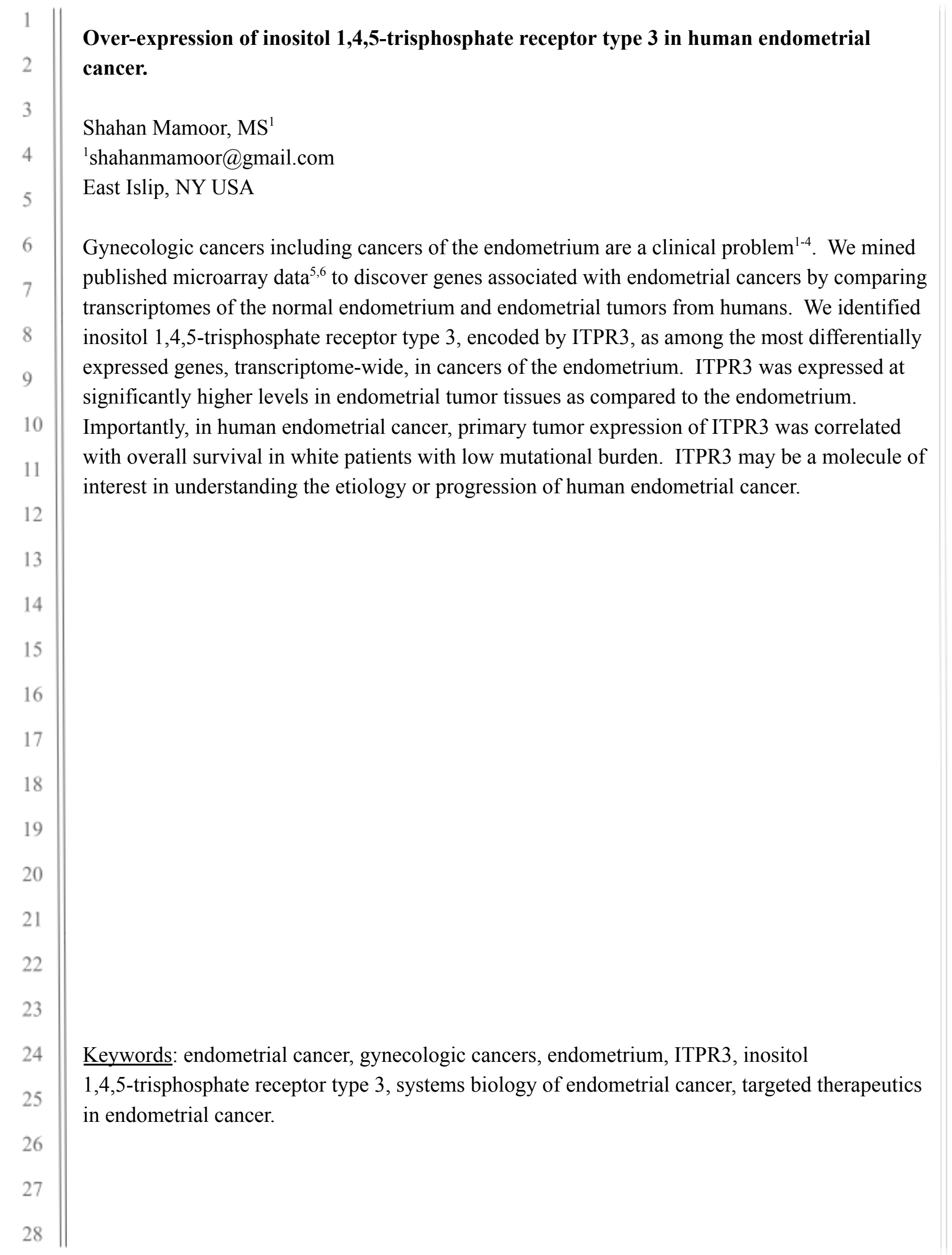


Endometrial cancer is the most common gynecologic cancer in the developed world ${ }^{1}$. Over the last three decades, the incidence of endometrial cancer has increased $21 \%{ }^{4}$ and the death rate has increased $100 \%{ }^{3}$. We harnessed the power of independently published microarray datasets $^{5,6}$ to determine in an unbiased fashion and at the systems-level genes most differentially expressed in endometrial tumors. We report here the differential and increased expression of the inositol 1,4,5-trisphosphate receptor type 3 (ITPR3) in human endometrial cancer.

\section{Methods}

We utilized datasets GSE63678 5 and GSE115810 ${ }^{6}$ for this global differential gene expression analysis of human endometrial cancer in conjunction with GEO2R. GSE63678 was generated using Affymetrix Human Genome U133A 2.0 Array technology with $n=5$ control endometrial tissues (including $n=4$ uterine myomas and $n=1$ benign cyst) and $n=7$ endometrial cancers (including $n=2$ endometrial adenocarcinomas, $n=3$ mixed endometrioid adenocarcinomas, and $n=2$ adenocarcinomas with squamous differentiation); analysis was performed using platform GPL571. GSE115810 was generated using Affymetrix Human Genome U133A Array technology with $n=3$ control endometrial tissues and $n=24$ endometrial cancers; analysis was performed using platform GPL96. The Benjamini and Hochberg method of $p$-value adjustment was used for ranking of differential expression but raw $p$-values were used to assess statistical significance of global differential expression. Log-transformation of data was auto-detected, and the NCBI generated category of platform annotation was used. A statistical test was performed to evaluate whether ITPR3 gene expression was significantly different between control endometrial tissue and endometrial tumor tissue in humans using a two-tailed t-test. For Kaplan-Meier survival analysis, we used the Kaplan-Meier plotter tool ${ }^{7}$ for correlation of ITPR3 mRNA expression levels with overall survival in $n=543$ endometrial cancer patients.

\section{$\underline{\text { Results }}$}

We harnessed the power of blind comparative transcriptome analysis using published microarray data ${ }^{5,6}$ to discover in an unbiased fashion genes associated with endometrial cancer in humans.

\section{ITPR3 is differentially expressed in endometrial cancer.}

We identified inositol 1,4,5-trisphosphate receptor type 3, encoded by ITPR3, as among the genes most differentially expressed in cancers of the endometrium when compared to benign endometrial tissues (Chart 1). When sorting each of the genes expressed in endometrial tumor tissue based on significance of change in expression as compared to benign endometrial tissue, ITPR3 ranked 139 out of 22273 transcripts, equating to $99.4 \%$ differential expression (Chart 1). Differential expression of ITPR3 in human endometrial cancers was statistically significant (Chart $1 ; p=1.52 \mathrm{E}-04)$. 
We queried a second microarray data to validate differential expression of ITPR3 in endometrial cancer. Again, we observed differential expression of ITPR3 when comparing endometrial tumor tissue to benign endometrial tissue (Chart 2). When sorting each of the genes expressed in endometrial tumor tissue based on significance of change in expression as compared to benign endometrial tissue, ITPR3 ranked 3030 out of 22283 transcripts, equating to $86.4 \%$ differential expression (Chart 2). Differential expression of ITPR3 in human endometrial cancers approached statistical significance (Chart $2 ; p=0.0554558)$.

\section{ITPR3 is expressed at significantly higher levels in endometrial cancers as compared to benign endometrial tissue.}

We obtained exact mRNA expression levels for ITPR3 in endometrial tumor tissues and from benign endometrial tissue to evaluate direction and statistical significance of change in expression of ITPR3 in human endometrial cancer. ITPR3 was expressed at higher levels in endometrial tissue as compared to normal endometrial tissue, and this difference was statistically significant (Figure $1 ; p=0.0011$ ). We calculated a mean fold change of 1.24 in ITPR3 mRNA levels in human endometrial cancer, as ITPR 3 was expressed at $8.33 \pm 0.73$ arbitrary units (A.U.) in control endometrial tissue but at $10.35 \pm 0.66$ A.U. in endometrial tumor tissue.

\section{ITPR3 expression is correlated with patient survival outcomes in endometrial cancer.}

We performed Kaplan-Meier survival analysis to evaluate correlation between ITPR3 primary tumor expression and survival outcomes in 543 patients with endometrial cancer. We observed a correlation between primary tumor expression of ITPR3 and overall survival in patients with endometrial cancer, in white patients with low mutational burden, in the upper survival tertile (Figure 2). ITPR3 primary tumor mRNA levels were a negative prognostic indicator in white endometrial cancer patients with low mutational burden. White patients with low mutational burden whose primary tumors expressed low levels of ITPR 3 possessed median OS of 103.73 months, while white patients with low mutational burden whose tumors expressed high levels of ITPR3 possessed median OS of 78.4 months. This difference in OS based on ITPR3 tumor expression in white patients with endometrial cancer with low mutational burden was statistically significant (Figure 2, Chart 3; logrank $p$-value: 0.02; hazard ratio: 2.18 (1.11-4.25)). ITPR3 primary endometrial tumor expression was not correlated with overall survival in white patients with high mutational burden (Figure 2, Chart 3; logrank $p$-value: 0.61; hazard ratio: 0.78 (0.31-1.98)), nor in black patients with high ((Figure 2, Chart 3; logrank p-value: 0.44 ; hazard ratio: $1.87(0.38-9.3)$ ) or low mutational burden (Figure 2, Chart 3; logrank p-value: 0.39 ; hazard ratio: $0.57(0.16-2.08))$.

Thus, by mining published microarray data ${ }^{5,6}$ in an unbiased and systematic fashion, we identified inositol 1,4,5-trisphosphate receptor type 3, encoded by ITPR3, as among the genes 


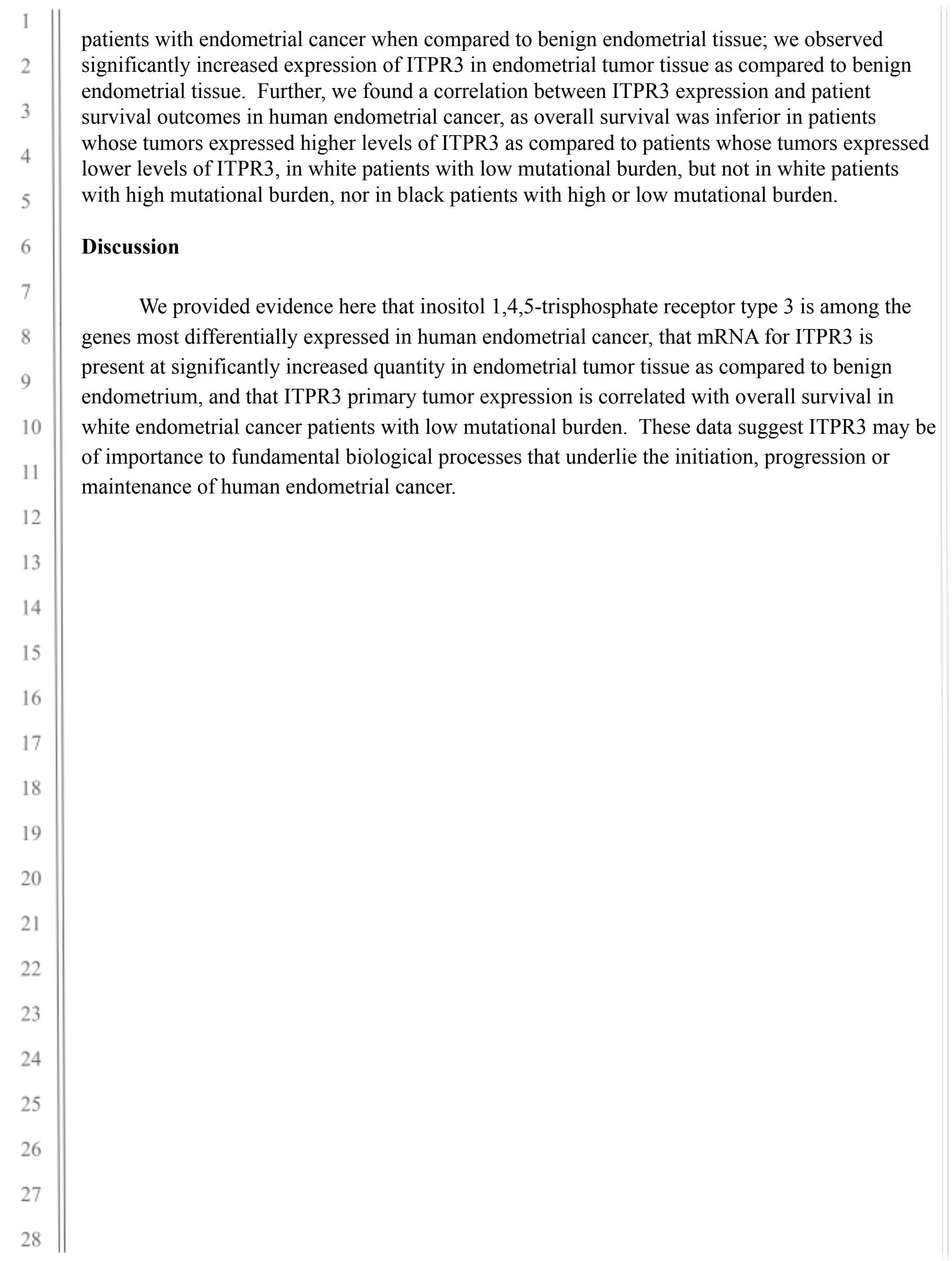




\section{References}

1. Amant, F., Moerman, P., Neven, P., Timmerman, D., Van Limbergen, E. and Vergote, I., 2005. Endometrial cancer. The Lancet, 366(9484), pp.491-505.

2. Sorosky, J.I., 2008. Endometrial cancer. Obstetrics \& Gynecology, 111(2), pp.436-447.

3. Morice, P., Leary, A., Creutzberg, C., Abu-Rustum, N. and Darai, E., 2016. Endometrial cancer. The Lancet, 387(10023), pp.1094-1108.

4. Sorosky, J.I., 2012. Endometrial cancer. Obstetrics \& Gynecology, 120(2 Part 1), pp.383-397.

5. Pappa, K.I., Polyzos, A., Jacob-Hirsch, J., Amariglio, N., Vlachos, G.D., Loutradis, D. and Anagnou, N.P., 2015. Profiling of discrete gynecological cancers reveals novel transcriptional modules and common features shared by other cancer types and embryonic stem cells. PLoS One, 10(11), p.e0142229.

6. Hermyt, E., Zmarzły, N., Grabarek, B., Kruszniewska-Rajs, C., Gola, J., Jęda-Golonka, A., Szczepanek, K., Mazurek, U. and Witek, A., 2019. Interplay between miRNAs and Genes Associated with Cell Proliferation in Endometrial Cancer. International journal of molecular sciences, 20(23), p.6011.

7. Nagy, A., Munkacsy, G. and Gyorffy, B., 2020. Pancancer survival analysis of cancer hallmark genes. bioRxiv. 
Rank: 139

2 Probe ID: 201189_s_at

p-value: $1.52 \mathrm{E}-04$

3 t: -5.4037036

$4 \quad$ B: 1.2537

Gene: ITPR3

5 Gene name: inositol 1,4,5-trisphosphate receptor type 3

Chart 1: ITPR3 is differentially expressed in endometrial cancer when comparing primary endometrial tumors to benign endometrial tissue.

The rank of global differential expression, probe/transcript ID, the $p$-value with respect to

9 differential expression transcriptome-wide, $t$, a moderated $t$-statistic, $\mathrm{B}$, the log-odds of differential expression between the groups compared, the gene and gene name are listed in this chart. 


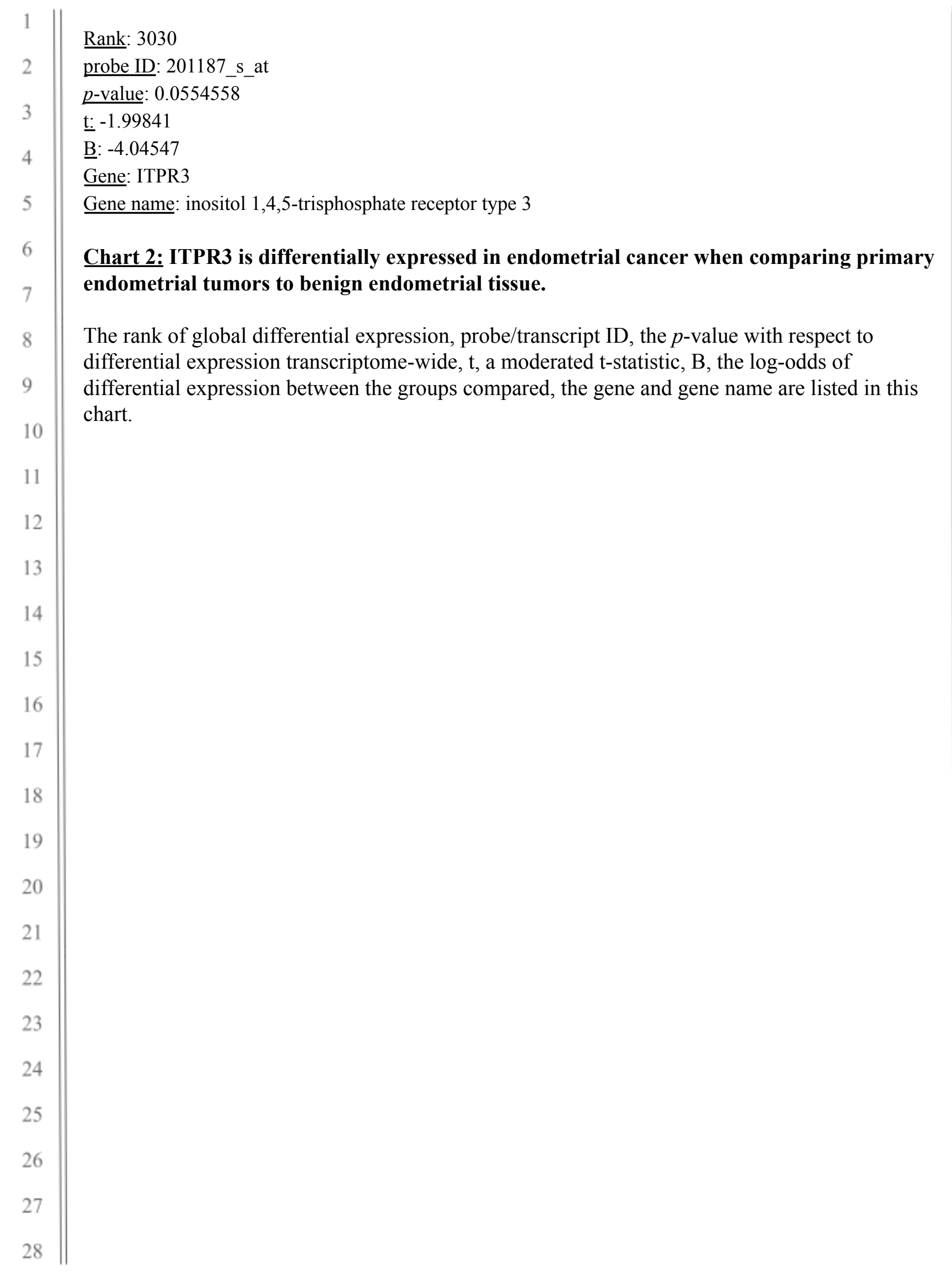




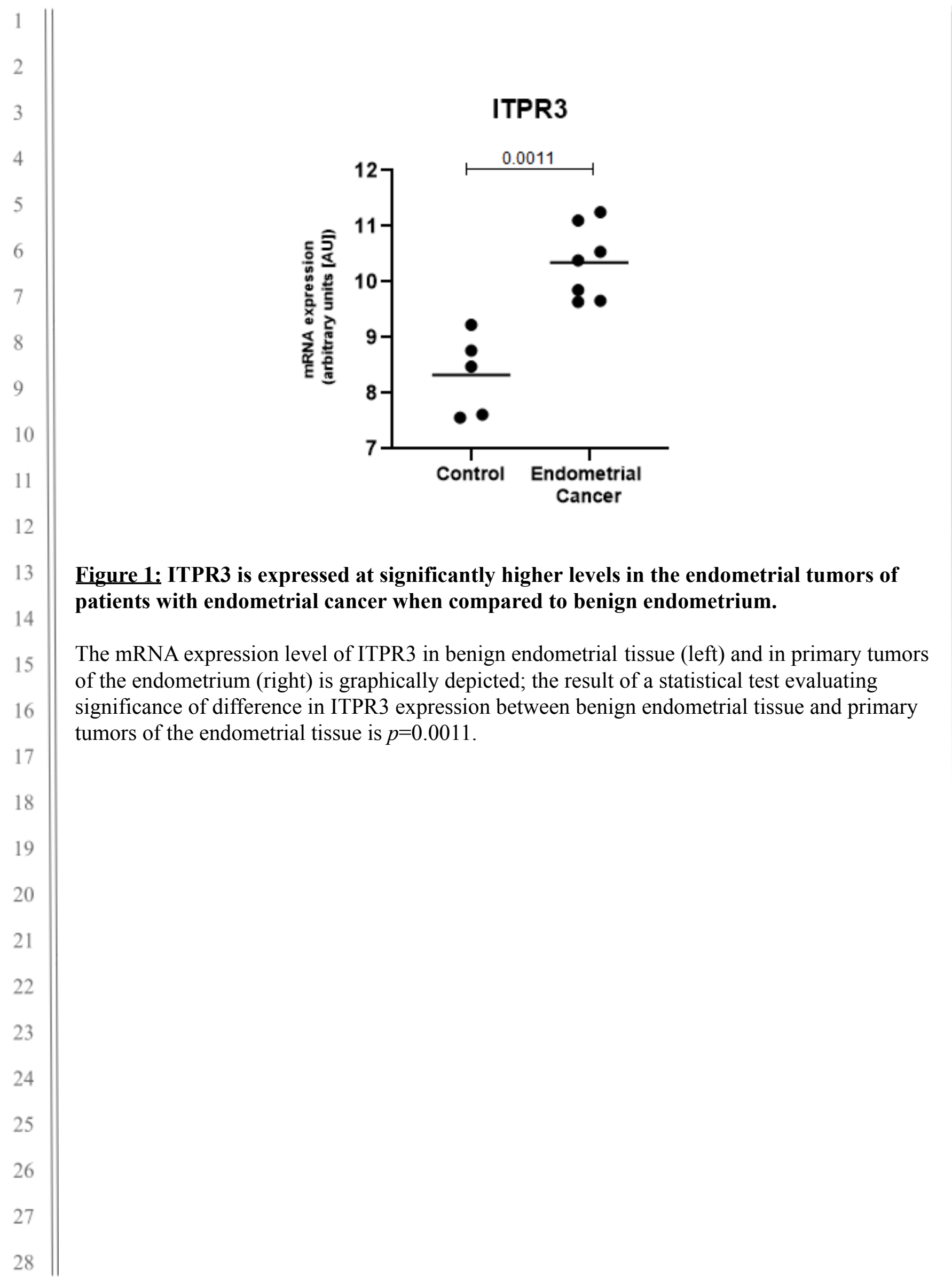




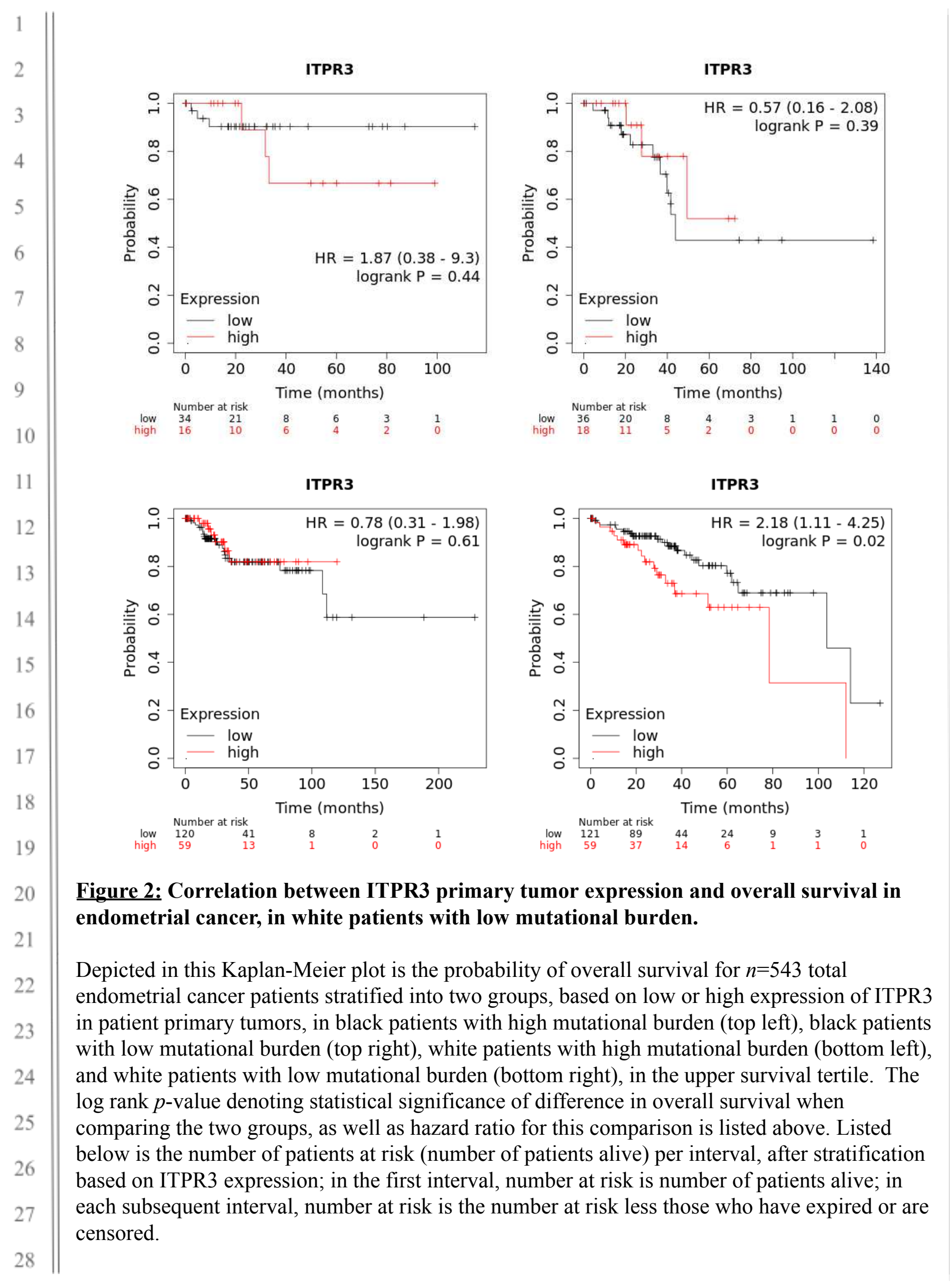


Low ITPR3 expression: 103.73 months

High ITPR3 expression: 78.4 months

Chart 3: Median overall survival is superior in endometrial cancer patients with low primary tumor expression of ITPR3, in white patients with low mutational burden.

The median OS (overall survival) of white endometrial cancer patients with low mutational

6 burden, with low primary tumor expression of ITPR3 and high primary tumor expression of ITPR3 is listed in this chart, in the upper survival tertile. 